\title{
The Classification of Differential Structures on Quantum 2-Spheres
}

\author{
Piotr Podleś * \\ RIMS, Kyoto University, Kyoto 606, Japan
}

Received March 13, 1992

\begin{abstract}
Exterior algebras of differential forms on quantum 2-spheres $S_{q c}^{2}$, $q \in[-1,1] \backslash\{0\}, c \in[0, \infty](c=0$ for $q= \pm 1)$, are classified. In the definition of exterior algebras we assume the invariance w.r.t. the action of the quantum $S U(2)$ group and "dimensionality conditions" (which imply that we deal with "twodimensional manifolds"). The exterior algebras exist only for $c=0$ and are unique in that case. The corresponding generalized directional derivatives are provided.
\end{abstract}

\section{Introduction}

One of the most important problems of theoretical physics is to find a consistent theory which would generalize both the general theory of relativity and quantum field theory. In the opinion of some physicists, in such a future theory functions on space-time should be replaced by operators belonging to a non-commutative algebra. In other words, space-time should be replaced by a quantum space. (The basic idea could be that the laws of physics should be the same in each quantum space-time.) Therefore, it is important to investigate the properties of quantum spaces, especially those properties which could be important for physics, like the existence of differential structures. In addition to general considerations (cf. e.g. [W 1, C, W 3, W 5, Mau]) we need also concrete examples (cf. e.g. [W 2, M, RTF, $\mathrm{PW}, \mathrm{CSSW}, \mathrm{CSW}, \mathrm{WZ}]$ ). One of them is given by the quantum spheres $S_{q c}^{2}$ [P 1], which are homogeneous spaces of quantum $S U(2)$ groups $S U_{q}(2)$ [W 2]. Quantum spheres are generalizations of the standard 2-sphere $S^{2}$ endowed with a classical right action of $S U(2)$ [or $S O(3)$ ]. (This action plays an important role in the description of spherical symmetric, stationary systems in physics, such as the hydrogen atom in quantum mechanics or the Schwarzschild solution in the general theory of relativity.)

* On leave from: Department of Mathematical Methods in Physics, Faculty of Physics, University of Warsaw, Hoża 74, PL 00-682 Warszawa, Poland 
The main result of the present paper is as follows:

1. The quantum sphere $S_{q 0}^{2}$ possesses a unique "two-dimensional $S U_{q}(2)$-invariant differential structure."

2. Quantum spheres $S_{q c}^{2}[q \in(-1,1) \backslash\{0\}, c>0]$ do not possess "two-dimensional $S U_{q}(2)$-invariant differential structures."

For the latter case it becomes interesting to consider "three-dimensional $S U_{q}(2)$ invariant differential structures." They are not classified yet, although a related example was presented in [P 2].

The main result is presented in Sect. 1. Its proof is given in Sect. 2. We should stress the following:

a) we overcome the difficulty related to the existence of a constraint in the left module of first order differential forms,

b) we describe differential forms of higher orders as well,

c) non-linearity of resulting commutation relations (6)-(8),

d) the full classification of considered objects.

In Sect. 3 we briefly study related generalized directional derivatives. The results of the paper were essentially contained in [P 3] and announced in [P 4].

In the following we sum over repeated indices (Einstein's convention). Throughout the paper we use the terminology and results of [W 2, W 3, P1, P 2]; we set $q \equiv \mu \in[-1,1] \backslash\{0\}, c \in[0, \infty]$ for $q \in(-1,1) \backslash\{0\}$ and $c=0$ for $q= \pm 1$. Moreover, $\mathscr{A} C C\left(S U_{q}(2)\right)$ is the *-algebra of polynomials on $S U_{q}(2)$ and $\Phi: \mathscr{A} \rightarrow \mathscr{A} \otimes \mathscr{A}, \kappa: \mathscr{A} \rightarrow \mathscr{A}, e: \mathscr{A} \rightarrow \mathbf{C}$ are the corresponding comultiplication, coinverse, and counit. Moreover, $\mathscr{A}_{c} \subset C\left(S_{q c}^{2}\right)$ is the *-algebra of polynomials on $S_{q c}^{2}$ generated by $e_{-1}, e_{0}, e_{1}$, and the $*$-homomorphism $\sigma_{q c}: \mathscr{A}_{c} \rightarrow \mathscr{A}_{c} \otimes \mathscr{A}_{\text {describes the }}$ action of $S U_{q}(2)$ on $S_{q c}^{2}$. The elements $e_{-1}, e_{0}, e_{1}$ satisfy the relations

$$
\begin{gathered}
e_{i}^{*}=e_{-i}, \quad i=-1,0,1, \\
a_{l m} e_{l} e_{m}=\varrho I, \\
b_{l m, k} e_{l} e_{m}=\lambda e_{k}, \quad k=-1,0,1,
\end{gathered}
$$

where the real numbers $a_{l m}, b_{l m, k}, \lambda, \varrho(l, m, k=-1,0,1)$ are given in $[\mathrm{P} 1,2 \mathrm{~b}-2 \mathrm{e}$ and Sect. 4]. Nonequivalent, irreducible, $(2 n+1)$-dimensional invertible representations of $S U_{q}(2)$ are denoted by $d_{n}, n=0,1 / 2,1, \ldots$ Then $d_{0}=(I)$ is the trivial representation, we choose $d_{1}=\left(d_{1, i j}\right)_{i, j=-1,0,1}$ as given in Sect. 2 of [P 1]. We have

$$
\sigma_{q c} e_{k}=e_{m} \otimes d_{1, m k}, \quad k=-1,0,1 .
$$

We embed (see [P1, Sect. 6]) $\mathscr{A}_{c}$ into $\mathscr{A}$ by the formula $e_{i}=s_{k} d_{1, k i}, i=-1,0,1$, where $\left(s_{-1}, s_{0}, s_{1}\right)$ equals $\left(c^{1 / 2}, 1, c^{1 / 2}\right)$ for $c<\infty$ and $(1,0,1)$ for $c=\infty$. Then $\sigma_{q c}=\Phi_{1 . \alpha_{c}}$.

\section{The Main Result}

Up to now there is no satisfactory functorial way of defining differential structures on quantum spaces. Therefore, we proceed in an axiomatic way. We first analyse some properties satisfied by differential forms on $S^{2}$. Then we classify differential structures on quantum spheres which satisfy these properties.

Let $\varrho$ be the standard right continuous action of $S U(2)$ on $S^{2} \approx U(1) \backslash S U(2)$ (we use the isomorphism described in Sect. 6 of [P1]) and $\sigma=\varrho^{*}: C\left(S^{2}\right)$ 
$\rightarrow C\left(S^{2}\right) \otimes C(S U(2))$ be the corresponding $C^{*}$-homomorphism. Moreover, we can define a right coaction $r$ of $S U(2)$ on $C\left(S^{2}\right)$ by formula

$$
\left(r_{g} f\right)(x)=f(\varrho(x, g)), \quad f \in C\left(S^{2}\right), \quad g \in S U(2), \quad x \in S^{2} .
$$

The last two mappings are related by $\left(\mathrm{id} \otimes \chi_{g}\right) \sigma=r_{g}$, where $g \in S U(2)$ and $\chi_{g}$ is the corresponding character on $C(S U(2))$. We know that $C(S U(2))=C\left(S U_{1}(2)\right), C\left(S^{2}\right)$ $=C\left(S_{10}^{2}\right), \sigma_{1 \infty_{0}}=\sigma_{10}$ with the identification $C\left(S_{10}^{2}\right) \ni e_{ \pm 1}= \pm i\left(x_{1} \pm i x_{2}\right) \in C\left(S^{2}\right)$, $C\left(S_{10}^{2}\right) \ni e_{0}=2 x_{3} \in C\left(S^{2}\right)$ (as in Sect. 3 of [P 2], we set radius of $S^{2}$ as $R=1 / 2$ ). Moreover, $S^{2}$ is a manifold, $\varrho$ is smooth and $\mathscr{A}_{0} \subset C^{\infty}\left(S^{2}\right)$ (the set of smooth functions on $S^{2}$ ).

We set $\mathscr{B}=\mathscr{A}_{0}$. Let $S^{\wedge}=\bigoplus_{n=0}^{\infty} S^{\wedge n}$, where

$$
S^{\wedge n}=\operatorname{span}\left\{a_{0} d a_{1} \wedge \ldots \wedge d a_{n}: a_{0}, a_{1}, \ldots, a_{n} \in \mathscr{B}\right\}
$$

is the bimodule of exterior differential forms on $S^{2}$ of $n^{\text {th }}$ degree, which are generated by $\mathscr{B}$. We denote the exterior derivative by $d: S^{\wedge} \rightarrow S^{\wedge}$. Let $*: S^{\wedge} \rightarrow S^{\wedge}$ be the complex conjugation:

$$
\left(a_{0} d a_{1} \wedge \ldots \wedge d a_{n}\right)^{*}=a_{0}^{*} d\left(a_{1}^{*}\right) \wedge \ldots \wedge d\left(a_{n}^{*}\right), \quad a_{0}, a_{1}, \ldots, a_{n} \in \mathscr{B} .
$$

We have moreover the right shifts $R_{g}: S^{\wedge} \rightarrow S^{\wedge}, g \in S U(2)$ :

$$
R_{g}\left(a_{0} d a_{1} \wedge \ldots \wedge d a_{n}\right)=\left(r_{g} a_{0}\right) d\left(r_{g} a_{1}\right) \wedge \ldots \wedge d\left(r_{g} a_{n}\right), \quad a_{0}, a_{1}, \ldots, a_{n} \in \mathscr{B} .
$$

Alternatively, we can also consider a unique linear mapping $\sigma^{\wedge}: S^{\wedge} \rightarrow S^{\wedge} \otimes \mathscr{A}$ such that (id $\left.\otimes \chi_{g}\right) \sigma^{\wedge}=R_{g}, g \in S U(2)$. It is easy to check that $\left(S^{\wedge}, \sigma^{\wedge}, d, *\right)$ satisfies

1) $S^{\wedge}=\bigoplus_{n=0}^{\infty} S^{\wedge n}$ is a graded algebra such that $S^{\wedge 0}=\mathscr{B}$ and the unity of $S^{\wedge 0}$ is the unity of $S^{\wedge}$.

2) $\sigma^{\wedge}: S^{\wedge} \rightarrow S^{\wedge} \otimes \mathscr{A}$ is a graded homomorphism such that

$$
(\mathrm{id} \otimes e) \sigma^{\wedge}=\mathrm{id}, \quad\left(\sigma^{\wedge} \otimes \mathrm{id}\right) \sigma^{\wedge}=(\mathrm{id} \otimes \Phi) \sigma^{\wedge}, \quad \sigma^{\wedge 0}=\sigma_{\mid \mathscr{B}} .
$$

3) * is a graded antilinear involution such that

$$
\left(\theta \wedge \theta^{\prime}\right)^{*}=(-1)^{k l} \theta^{*} \wedge \theta^{*}, \quad \theta \in S^{\wedge k}, \quad \theta^{\prime} \in S^{\wedge l}
$$

$\left(\wedge\right.$ denotes multiplication in $\left.S^{\wedge}\right)$,

$$
\left(\sigma^{\wedge}\right) *=(* \otimes *) \sigma^{\wedge},
$$

* on $S^{\wedge 0}$ reduces itself to the standard *.

4) $d: S^{\wedge} \rightarrow S^{\wedge}$ is a linear mapping such that

a) $d\left(S^{\wedge n}\right) \subset S^{\wedge(n+1)}, n=0,1,2, \ldots$,

b) $d\left(\theta \wedge \theta^{\prime}\right)=d \theta \wedge \theta^{\prime}+(-1)^{k} \theta \wedge d \theta^{\prime}, \theta \in S^{\wedge k}, \theta^{\prime} \in S^{\wedge}$,

c) $d *=* d$,

d) $(d \otimes \mathrm{id}) \sigma^{\wedge}=\sigma^{\wedge} d$,

e) $d d=0$.

5) $S^{\wedge n}=\operatorname{span}\left\{a_{0} d a_{1} \wedge \ldots \wedge d a_{n}: a_{0}, a_{1}, \ldots, a_{n} \in \mathscr{B}\right\}$ (we omit $\wedge$ if one of multipliers belongs to $S^{\wedge 0}$ ).

In the following we assume that $q \in[-1,1] \backslash\{0\}, c \in[0, \infty](c=0$ for $q= \pm 1)$ and $\mathscr{A} \subset C\left(S U_{q}(2)\right)$ is the *-algebra of polynomials on $S U_{q}(2), \mathscr{B}=\mathscr{A}_{c} \subset C\left(S_{q c}^{2}\right)$, $\sigma=\sigma_{q c}$. 
Definition 1. We say that $\mathbf{S}^{\wedge}=\left(S^{\wedge}, \sigma^{\wedge}, d, *\right)$ is an exterior algebra on $S_{q c}^{2}$, invariant w.r.t. $\sigma_{q c}$ iff conditions 1)-5) are satisfied.

The above choice of axioms is motivated by [C, W 5]. We don't introduce (and don't know if it is in a self-consistent way possible) any conditions replacing the classical condition

$$
\theta \wedge \theta^{\prime}=(-1)^{k l} \theta^{\prime} \wedge \theta, \quad \theta \in S^{\wedge k}, \quad \theta^{\prime} \in S^{\wedge l},
$$

which does not hold in the case of a non-commutative $\mathscr{B}$. Instead, we can introduce "dimensionality" conditions as follows. Let $\mathbf{S}^{\wedge}$ be as in Definition 1 and $P=a_{k l} e_{k} d e_{l}\left[a_{k l}\right.$ were used in (2)]. Using (5) of [P 2] we get $\sigma^{\wedge 1} P=P \otimes I$, i.e. $P$ is $\sigma^{\wedge 1}$-invariant. Moreover, it is easy to check that $P$ is unique (up to a scalar) $\sigma^{\wedge 1}$-invariant element of $\mathscr{A}_{c} \cdot \operatorname{span}\left\{d e_{-1}, d e_{0}, d e_{1}\right\}$. Let now $q=1$. Then $P \equiv 4 x_{k} d x_{k}$. Consider the exterior algebra $S^{\wedge}$ given at the beginning of the present section. In that case $d x_{k}, k=1,2,3$, generate the left module $S^{\wedge 1}$ with only one constraint, namely $P=0$. In terms of $e_{k}, k=-1,0,1$, it means that

6) $d e_{k}, k=-1,0,1$, generate the left module $S^{\wedge 1}\left(\right.$ over $\left.S^{\wedge 0}=\mathscr{A}_{c}\right)$.

7) For any $a_{k} \in \mathscr{A}_{c}, k=-1,0,1$,

$$
a_{k} d e_{k}=0 \Leftrightarrow \exists a \in \mathscr{A}_{c}: \quad a_{k}=a \cdot a_{m k} e_{m}, \quad k=-1,0,1 .
$$

Moreover, considering $\varepsilon_{i j k} x_{i} d x_{j} \wedge d x_{k} \in S^{\wedge 2}$, where $\varepsilon_{i j k}, i, j, k=1,2,3$, is the completely antisymmetric symbol with $\varepsilon_{123}=1$, one obtains (with the basis given by the above element)

8) there exists a one-element $\sigma^{\wedge 2}$-invariant basis of the left module $S^{\wedge 2}$.

Definition 2. Let $\mathbf{S}^{\wedge}=\left(S^{\wedge}, \sigma^{\wedge}, d, *\right)$ be an exterior algebra on $S_{q c}^{2}$, invariant w.r.t. $\sigma_{q c}$. We say that $\mathbf{S}^{\wedge}$ is $\left({ }^{2}\right)$-dimensional iff conditions 6$\left.)-8\right)$ are satisfied.

Remark. Symbol $\left({ }^{2}\right)$ reminds us that the left modules $S^{\wedge n}$ are (in some sense) $\left(\begin{array}{l}2 \\ n\end{array}\right)$-dimensional, $n=0,1,2$.

Theorem. For $q \in[-1,1] \backslash\{0\}, c=0$ there exists a unique $\left({ }^{2}\right)$-dimensional exterior algebra $\mathbf{S}^{\wedge}$ on the quantum sphere $S_{q c}^{2}$, invariant w.r.t. $\sigma=\sigma_{q c}$.

For $q \in(-1,1) \backslash\{0\}, c \in(0, \infty]$ there are no $\left(^{2}\right)$-dimensional exterior algebras on the quantum spheres $S_{q c}^{2}$, invariant w.r.t. $\sigma=\sigma_{q c}$.

The same facts hold if we restrict ourselves to $S^{\wedge 0} \oplus \ldots \oplus S^{\wedge k}$ for some $k=1,2, \ldots$ (with suitable restrictions of all structures in $\mathbf{S}^{\wedge}$, without $*$ or with $*$ ), instead of $\mathbf{S}^{\wedge}$ (in Definitions 1-2 and in this theorem).

Moreover, for $c=0 \mathbf{S}^{\wedge}$ has the following properties:

a) one-element $\sigma^{\wedge 2}$-invariant basis in the left module $S^{\wedge 2}$ can be chosen as

$$
\omega=a_{k l} e_{k} b_{m n, l} d e_{m} \wedge d e_{n},
$$

b) $S^{\wedge k}=\{0\}, k>2$,

c) the following formulae hold:

$$
\begin{gathered}
a_{k l}\left(d e_{k}\right) e_{l}=0, \\
b_{k l, r}\left(d e_{k}\right) e_{l}=\left(1-q^{2}\right) d e_{r}-b_{k l, r} e_{k} d e_{l}, \quad r=-1,0,1, \\
c_{k l, r}\left(d e_{k}\right) e_{l}=c_{k l, r} e_{k}\left[d e_{l}+q^{-2}\left(1-q^{2}\right) b_{m n, l} e_{m} d e_{n}\right], \quad r=-2, \ldots, 2, \\
\omega e_{r}=e_{r} \omega, \quad r=-1,0,1, \\
a_{k l} d e_{k} \wedge d e_{l}=0,
\end{gathered}
$$




$$
\begin{gathered}
b_{k l, r} d e_{k} \wedge d e_{l}=e_{r} \omega, \quad r=-1,0,1, \\
c_{k l, r} d e_{k} \wedge d e_{l}=q^{-2}\left(1+q^{2}\right)^{-2}\left(q^{6}-1\right) c_{k l, r} e_{k} e_{l} \omega, \quad r=-2, \ldots, 2, \\
\left(d e_{k}\right)^{*}=d e_{-k}, \quad k=-1,0,1, \\
\omega^{*}=-\omega, \\
\sigma^{\wedge 1} d e_{k}=d e_{m} \otimes d_{1, m k}, \quad k=-1,0,1, \\
\sigma^{\wedge 2} \omega=\omega \otimes I
\end{gathered}
$$

$\left(a_{l m}, b_{l m, k}, c_{l m, k}, l, m=-1,0,1\right.$, were used in (2), (3), and [P 2, Eq. (4)]).

Remark. According to [W 4, p. 75], $S U_{q}(2), q \in[-1,1] \backslash\{0\}$, give all compact matrix quantum groups which have the same representation theory as $S U(2)$. Next, quantum spheres $\left(S_{q c}^{2}, \sigma_{q c}\right), c \in[0, \infty]$ for $q \in(-1,1) \backslash\{0\}$ and $c=0$ for $q= \pm 1$, give all generalizations of $S^{2}$ endowed with the standard right action of $S U(2)$ (Theorem 2 and Remarks $2-3$ of [P 1]; in the case of $q=-1$ see also [P 5]). Therefore, the above theorem classifies all generalizations of the exterior algebra of differential forms on $S^{2}$ (we consider only forms which are generated by the Cartesian coordinates).

\section{Proof of the Theorem}

For $q=1$ all conditions are satisfied by the exterior algebra introduced at the beginning of the previous section, hence the existence follows. The theorem holds in this case [proof of uniqueness is similar as for $q \in(-1,1) \backslash\{0\}-$ see below]. The case $(q, c)=(-1,0)$ can be reduced to the case $(q, c)=(1,0)$ (see $[\mathrm{P} 1$, Remark 3 after Theorem 2] and [P 5]). In the following we investigate the case $q \in(-1,1) \backslash\{0\}$.

We start with the following remarks. Set $M=a \dot{\oplus} b \dot{\oplus} c$ (the first column of the matrix $M$ is given by the matrix $a$, the next three columns of $M$ coincide with the successive columns of the matrix $b$ and the last five columns of $M$ coincide with the columns of $c$ ). Due to (5) of [P 2], $M$ intertwines $d_{0} \oplus d_{1} \oplus d_{2}$ with $d_{1} \oplus d_{1}$. Since $a, b, c$ are non-zero, $M$ is invertible. Therefore, $M^{-1}$ exists and intertwines $d_{1} \oplus d_{1}$ with $d_{0} \oplus d_{1} \oplus d_{2}$. Denote the matrix elements of $M^{-1}$ by $A_{k l}, B_{r, k l}(r=-1,0,1)$, $C_{r, k l}(r=-2, \ldots, 2), k, l=-1,0,1$. Then

$$
A\left(B , C \text { , resp.) intertwines } d _ { 1 } \text { (1) } d _ { 1 } \text { with } d _ { 0 } \left(d_{1}, d_{2}\right.\right. \text {, resp.). }
$$

It is easy to see that $w=\left(d_{1}^{T}\right)^{-1}$ is an invertible representation of $S U_{q}(2)$ $\left(w=\overline{\left[(\mathrm{id} \otimes \kappa)\left(\overline{d_{1}}\right)\right]^{T}}\right)$. By virtue of $(17)$ we get

$$
\left(d_{1}\right)_{0 m} B_{m, k l}=B_{0, a b}\left(d_{1}^{T}\right)_{k a}\left(d_{1}^{T}\right)_{l b}, \quad k, l=-1,0,1 .
$$

Multiplying both sides from the left by $w_{t l} w_{s k}$ one obtains

$$
w_{t l} w_{s k} B_{m, k l} d_{1,0 m}=B_{0, s t} I, \quad s, t=-1,0,1 \text {. }
$$

Analogously,

$$
w_{t l} w_{s k} C_{m, k l} d_{2,0 m}=C_{0, s t} I, \quad s, t=-1,0,1 .
$$

The equation $M^{-1} M=1$ gives

$$
\left.\begin{array}{rl}
a_{k l} B_{r, k l} & =0, \quad a_{k l} C_{r, k l}=0, \quad c_{k l, r^{\prime}} C_{r, k l}=\delta_{r r^{\prime}}, \\
b_{k l, r^{\prime}} B_{r, k l} & =\delta_{r r^{\prime}}, \quad c_{k l, r^{\prime}} B_{r, k l}=0, \quad b_{k l, r^{\prime}} C_{r, k l}=0,
\end{array}\right\}
$$


for all possible $r, r^{\prime}$. On the other hand, $M M^{-1}=1$ is equivalent to

$$
A_{i j} a_{k l}+B_{r, i j} b_{k l, r}+C_{r, i j} c_{k l, r}=\delta_{i k} \delta_{j l},
$$

$i, j, k, l=-1,0,1$. After easy computations one can obtain

$$
\begin{gathered}
A_{-11}=q^{4}\left(1+q^{2}\right)^{-1}\left(1+q^{2}+q^{4}\right)^{-1}, \quad A_{00}=q^{2}\left(1+q^{2}+q^{4}\right)^{-1}, \\
A_{1-1}=q^{2}\left(1+q^{2}\right)^{-1}\left(1+q^{2}+q^{4}\right)^{-1}, \\
B_{-2,-1-1}=0, \quad B_{-1,-10}=-q^{2}\left(1+q^{4}\right)^{-1}, \quad B_{-1,0-1}=\left(1+q^{4}\right)^{-1}, \\
B_{0,-11}=q^{2}\left(1+q^{2}\right)^{-1}\left(1+q^{4}\right)^{-1}, \quad B_{0,00}=\left(1-q^{2}\right)\left(1+q^{4}\right)^{-1}, \\
B_{0,1-1}=-q^{2}\left(1+q^{2}\right)^{-1}\left(1+q^{4}\right)^{-1}, \quad B_{1,01}=-q^{2}\left(1+q^{4}\right)^{-1}, \\
B_{1,10}=\left(1+q^{4}\right)^{-1}, \quad B_{2,11}=0, \\
C_{-2,-1-1}=1, \quad C_{-1,-10}=\left(1+q^{4}\right)^{-1}, \quad C_{-1,0-1}=q^{2}\left(1+q^{4}\right)^{-1}, \\
C_{0,-11}=-q^{2}\left(1+q^{4}\right)^{-1}\left(1+q^{2}+q^{4}\right)^{-1}, \\
C_{0,00}=q^{2}\left(1+q^{2}\right)^{2}\left(1+q^{4}\right)^{-1}\left(1+q^{2}+q^{4}\right)^{-1}, \\
C_{0,1-1}=-q^{6}\left(1+q^{4}\right)^{-1}\left(1+q^{2}+q^{4}\right)^{-1}, \quad C_{1,01}=\left(1+q^{4}\right)^{-1}, \\
C_{1,10}=q^{2}\left(1+q^{4}\right)^{-1}, \quad C_{2,11}=1, \\
A_{k l}=0 \quad \text { for } \quad k+l \neq 0, \\
B_{r, k l}=0, \quad C_{r, k l}=0 \quad \text { for } \quad k+l \neq r .
\end{gathered}
$$

Note. Equation (5) of [P 2] and the above remarks are true also for $q= \pm 1$. In that case $\tilde{e}_{r}$ are defined as in [P 2, Eq. (4)] (i.e. $\left.\tilde{e}_{r}=c_{l m, r} e_{l} e_{m}, r=-2, \ldots, 2\right)$ with $c_{l m, r}$ given in the preceding formulae, while $d_{2}$ can be determined from [P2, Eq. (5)] for $q= \pm 1$.

Proof of Uniqueness. Let $\mathbf{S}^{\wedge}=\left(S^{\wedge}, \sigma^{\wedge}, d, *\right)$ be $\left({ }^{2}\right)$-dimensional exterior algebra on the quantum sphere $S_{q c}^{2}$, invariant w.r.t. $\sigma_{q c}, q \in(-1,1) \backslash\{0\}, c \in[0, \infty]$. Using condition 4.b, (2)-(3), and condition 7 we obtain

$$
\begin{gathered}
a_{k l}\left(d e_{k}\right) e_{l}=d\left(a_{k l} e_{k} e_{l}\right)-a_{k l} e_{k}\left(d e_{l}\right)=\varrho d I-0=0, \\
b_{k l, r}\left(d e_{k}\right) e_{l}=d\left(b_{k l, r} e_{k} e_{l}\right)-b_{k l, r} e_{k}\left(d e_{l}\right)=\lambda d e_{r}-b_{k l, r} e_{k} d e_{l} .
\end{gathered}
$$

We set $\theta_{r}=c_{k l, r}\left(d e_{k}\right) e_{l}, r=-2, \ldots, 2$. Using condition 2, condition 4.d, and [P 2, Eq. (5)], we get $\sigma^{\wedge} \theta_{r}=\theta_{m} \otimes d_{2, m r}, r=-2, \ldots, 2$. The analogous fact holds also for $\theta_{r}^{\prime}=c_{k l, r} e_{k}\left(d e_{l}\right)$ and for $\theta_{r}^{\prime \prime}=c_{k l, r} e_{k} b_{m n, l} e_{m} d e_{n}$. According to conditions 6 and 7,

$$
S^{\wedge 1} \approx \mathscr{A}_{c} \otimes \operatorname{span}\left\{e_{-1}, e_{0}, e_{1}\right\} / \mathscr{A}_{c}\left(a_{m n} e_{m} \otimes e_{n}\right)
$$

as far as the transforming properties w.r.t. $S U_{q}(2)$ are concerned. But the numerator of the last expression transforms according to

$$
\left(d_{0} \oplus d_{1} \oplus \ldots\right) \oplus d_{1} \approx d_{0} \oplus 3 d_{1} \oplus 3 d_{2} \oplus \ldots,
$$

while the denominator according to $d_{0} \oplus d_{1} \oplus d_{2} \oplus \ldots$. Therefore, in a decomposition of $S^{\wedge 1}$ into a direct sum of vector subspaces, which correspond to invertible irreducible representations of $S U_{q}(2)$, there are exactly 2 subspaces corresponding to $d_{2}$. Thus, since $\theta_{2}^{\prime}$ and $\theta_{2}^{\prime \prime}$ are linearly independent,

$$
\theta_{r}=h \theta_{r}^{\prime}+b \theta_{r}^{\prime \prime}, \quad r=-2, \ldots, 2,
$$


for some $h, b \in \mathbf{C}$. The above equalities and (21) yield

$$
\left(d e_{i}\right) e_{j}=\lambda B_{r, i j}\left(d e_{r}\right)-B_{r, i j} b_{k l, r} e_{k} d e_{l}+C_{r, i j} c_{k l, r} e_{k}\left[h \cdot d e_{l}+b \cdot b_{m n, l} e_{m} d e_{n}\right],
$$

$i, j=-1,0,1$.

Due to conditions 6-7, for each $a \in \mathscr{A}_{c}$ there exist unique elements $R_{k l}(a) \in \mathscr{A}_{c}$, $k, l=-1,0,1$, such that $\left(d e_{k}\right) a=R_{k l}(a) d e_{l}, R_{k l}(a) e_{l}=0, k=-1,0,1$. Using (23) and (2)-(3) one can easily get

$$
\left.\begin{array}{rl}
R_{i n}\left(e_{j}\right)= & \lambda B_{n, i j} I-B_{r, i j} b_{k n, r} e_{k} \\
& +C_{r, i j} c_{k l, r} e_{k}\left[h \cdot \delta_{l n} I+b \cdot b_{m n, l} e_{m}-\varrho^{-1}(h+\lambda b) e_{l} a_{s n} e_{s}\right]
\end{array}\right\}
$$

$R_{i n}(I)=\delta_{i n} I-\varrho^{-1} e_{i} a_{s n} e_{s}, i, n, j=-1,0,1$. We define $r_{i n}$ as the superposition of the counit $e$ with $R_{i n}, r_{i n}=e \circ R_{i n}, i, n=-1,0,1$. Then

$$
\begin{gathered}
r_{i n}\left(e_{j}\right)=\lambda B_{n, i j}-B_{r, i j} b_{k n, r} s_{k} \\
+C_{r, i j} c_{k l, r} s_{k}\left[h \cdot \delta_{l n}+b \cdot b_{m n, l} s_{m}-\varrho^{-1}(h+\lambda b) s_{l} a_{t n} s_{t}\right], \\
r_{i n}(I)=\delta_{i n}-\varrho^{-1} s_{i} a_{t n} s_{t} .
\end{gathered}
$$

Since $R_{i j}(x) R_{j k}(y)=R_{i k}(x y), i, k=-1,0,1, x, y \in \mathscr{A}_{c}$ (the left-hand side satisfies conditions defining the right-hand side),

$$
r_{i j}(x) r_{j k}(y)=r_{i k}(x y) \text {. }
$$

Specializing $x=I, y=e_{m}$, we obtain

$$
a_{s j} e_{s} R_{j k}\left(e_{m}\right)=0, \quad k, m=-1,0,1,
$$

and $a_{t j} s_{t} r_{j k}\left(e_{m}\right)=0$. Considering the latter equation for $(m, k)=(1,-1)$ and $(1,0)[$ for $c=0:(m, k)=(-1,-1)$ and $(1,1)]$ we obtain

$$
h=\frac{q^{6}+q^{2}\left(1+q^{6}\right)\left(1+q^{2}\right) c}{q^{6}+\left(1+q^{6}\right)^{2} c}, \quad b=\frac{q^{4}\left(1-q^{2}\right)}{q^{6}+\left(1+q^{6}\right)^{2} c}
$$

[for $c=\infty: h=q^{2}\left(1+q^{2}\right)\left(1+q^{6}\right)^{-1}, b=0$ ]. Inserting these data into the equation

$$
r_{-1 k}\left(e_{1}\right) r_{k-1}\left(e_{0}\right)-q^{2} r_{-1 k}\left(e_{0}\right) r_{k-1}\left(e_{1}\right)=\lambda r_{-1-1}\left(e_{1}\right)
$$

[we obtain it by acting with $r_{-1-1}$ on both sides of (3) for $k=1$ and using (25)] one can easily get $c=0$ (it proves the second statement of the theorem). Hence [see (22) and the preceding formulae ], (6)-(8) are satisfied. It determines uniquely $\wedge, d, \sigma^{\wedge}$, and $*$ on the level of $S^{\wedge 0} \oplus S^{\wedge 1}$.

Acting $d$ on both sides of $a_{k l} e_{k} d e_{l}=0$, we get (10) (see conditions 4.b, 4.e). We shall now prove the property a). Let the matrix $t$ be a nonzero intertwinner of $d_{0}$ with $d_{2} \oplus d_{2}$ :

$$
d_{2, m k} d_{2, n s} t_{k s}=t_{m n} I, \quad m, n=-2, \ldots, 2 .
$$

Then $z=t_{k l} \tilde{e}_{k} \tilde{e}_{l} \in \mathbf{C I}$ (see [P 2, Eq. (4)]; we use $\sigma z=z \otimes I$ ). Multiplying $t$ by a factor, we can assume $z=I$ or $z=0$. Assume for the moment that $\omega=0$ [see (5)]. Considering the transforming properties and using [P 2, Eq. (5)] it is easy to see that a $\sigma^{\wedge 2}$-invariant basis $\Omega$ of the left module $S^{\wedge 2}$ must have a form

$$
\Omega=\lambda_{1} a_{m n} d e_{m} \wedge d e_{n}+\lambda_{2} a_{k l} e_{k} b_{m n, l} d e_{m} \wedge d e_{n}+\lambda_{3} t_{k l} \tilde{e}_{k} c_{m n, l} d e_{m} \wedge d e_{n},
$$


with some $\lambda_{1}, \lambda_{2}, \lambda_{3} \in \mathbf{C}$. But the first two components are now zero and we can assume $\lambda_{3}=1$. The transforming properties give ( $\Omega$ is a basis)

$$
\begin{array}{ll}
b_{m n, l} d e_{m} \wedge d e_{n}=a \cdot e_{l} \Omega, & l=-1,0,1, \\
c_{m n, l} d e_{m} \wedge d e_{n}=b \cdot \tilde{e}_{l} \Omega, & l=-1,0,1,
\end{array}
$$

for some $a, b \in \mathbf{C}$. Multiplying (*) from left by $a_{k l} e_{k}$, we get $0=\omega=a \Omega, a=0$. Multiplying (**) from the left by $t_{k l} \tilde{e}_{k}$ we obtain $\Omega=b z \Omega$. Therefore, $z \neq 0, z=I$, $b=1$. Using (21), one has

$$
d e_{i} \wedge d e_{0}=A_{i 0} a_{m n} d e_{m} \wedge d e_{n}+B_{r, i 0} b_{m n, r} d e_{m} \wedge d e_{n}+C_{r, i 0} c_{m n, r} d e_{m} \wedge d e_{n} .
$$

Combining this with condition $7,(10)$, and $(*)-(* *)$, one gets

$$
0=a_{k i} e_{k} d e_{i} \wedge d e_{0}=a_{k i} e_{k} C_{r, i 0} \tilde{e}_{r} \Omega,
$$

$a_{k i} e_{k} C_{r, i 0} \tilde{e}_{r}=0$. Applying the counit $e$, we obtain the contradiction $C_{0,00}=0$. Therefore, $\omega \neq 0$ and property a) easily follows ( $\omega$ is $\sigma^{\wedge 2}$-invariant). By an argument similar to that in the previous reasoning we obtain (11) and

$$
c_{k l, r} d e_{k} \wedge d e_{l}=y \tilde{e}_{r} \omega, \quad r=-2, \ldots, 2,
$$

for some $y \in \mathbf{C}$. Then the equation $0=a_{k i} e_{k} d e_{i} \wedge d e_{0}$ yields (cf. the previous considerations) $B_{0,00}+y C_{0,00}=0$. This and (27) give (12). All structures on the level of $S^{\wedge 0} \oplus S^{\wedge 1} \oplus S^{\wedge 2}$ are now determined uniquely.

Due to the transforming properties, $\omega e_{r}=\varepsilon \cdot e_{r} \omega, r=-1,0,1$, for some $\varepsilon \in \mathbf{C}$. Therefore, $0=\omega\left[b_{k l, r} e_{k} e_{l}-\lambda e_{r}\right]=\left[\varepsilon^{2} b_{k l, r} e_{k} e_{l}-\lambda \varepsilon e_{r}\right] \omega=\lambda\left(\varepsilon^{2}-\varepsilon\right) e_{r} \omega, \quad r=-1,0,1$. Hence $\varepsilon=1\left(\varepsilon=0\right.$ leads to the contradiction $\left.\omega \varrho=\omega a_{m n} e_{m} e_{n}=0, \omega=0\right)$ and (9) follows [for $q=1$ (9) would follow from the fact that the exterior algebra considered in the previous section is unique on the level of $\left.S^{\wedge 0} \oplus S^{\wedge 1} \oplus S^{\wedge 2}\right]$. Due to (10), (21), $(10-12)$, and (9) we get

$$
\left.\begin{array}{rl}
0 & =d e_{k} \wedge d e_{m} \wedge a_{m n} d e_{n} \\
& =\omega \wedge a_{m n}\left[B_{r, k m} e_{r}+y C_{r, k m} \tilde{e}_{r}\right] d e_{n} \\
& =\omega \wedge\left[\varrho_{1} b_{m n, k} e_{m} d e_{n}+\varrho_{2} d e_{k}\right], \quad k=-1,0,1,
\end{array}\right\}
$$

where $y=q^{-2}\left(1+q^{2}\right)^{-2}\left(q^{6}-1\right), \quad \varrho_{1}=\left[1+y\left(q^{2}-1\right)\right]\left(1+q^{4}\right)^{-1}, \quad \varrho_{2}=-y q^{2}$ $\times\left(1+q^{2}+q^{4}\right)^{-1}$ (in order to prove the last equality in (28), one can check by direct computation that

$$
a_{m n}\left[B_{r, k m} e_{r}+y C_{r, k m} \tilde{e}_{r}\right]=\varrho_{1} b_{m n, k} e_{m}+\varrho_{2} \delta_{k n}+y e_{k} a_{m n} e_{m},
$$

$n=-1,0,1, k=1$, multiply both sides from the right by $d e_{n}$, use condition 7 and the transforming properties). Multiplying both sides of (28) by $b_{s k, l} e_{s}$, using (9),

$$
b_{s k, l} e_{s} b_{m n, k} e_{m} d e_{n}=\left(1-q^{2}\right) b_{m n, s} e_{m} d e_{n}+q^{2} d e_{s}
$$

(it suffices to check it for $s=1$ and use the transforming properties) and comparing the obtained result with (28), one gets $\omega \wedge d e_{k}=0, k=-1,0,1$. This proves the property b). Hence, the uniqueness follows.

Proof of Existence. In Sect. 1 of [P 2] the exterior algebras $\Gamma_{c}^{\wedge}=\left(\Gamma_{c}^{\wedge}, \Gamma_{c} \Phi^{\wedge}, d, *\right)$ on $S_{q c}^{2}$, invariant w.r.t. $\sigma_{q c}$, were described $\left({ }_{\Gamma_{c}} \Phi^{\wedge}{ }_{\Gamma_{\Gamma}} \Phi^{\wedge}{ }_{\Gamma_{c_{c}}}\right)$. Let us consider the element $\tau=a_{k l} e_{k} d e_{l} \in \Gamma_{0}^{\wedge 1}$. According to [P 2, Eq. (8)],

$$
\tau=\text { (non-zero factor) } \cdot\left(-q e_{-1} \omega_{1}+q^{-2} e_{1} \omega_{-1}-e_{0} \Gamma+q\left(1+q^{2}\right)^{-1}\left(I-e_{0}\right) \xi\right) \text {. }
$$


Using [P 2, Eq. (6)] one can easily get $\tau e_{1}=e_{1} \tau$. But

$$
\Gamma_{0} \Phi^{\wedge}\left(\tau e_{1}\right)=\tau e_{k} \otimes d_{1, k 1}, \quad \Gamma_{0} \Phi^{\wedge}\left(e_{1} \tau\right)=e_{k} \tau \otimes d_{1, k 1} .
$$

Hence, $\tau e_{k}=e_{k} \tau, k=-1,0,1$. Moreover,

$$
\tau^{*}=a_{k l}\left(d e_{-\imath}\right) e_{-k}=a_{l k}\left(d e_{l}\right) e_{k}=-\tau
$$

[we used (1)]. Using these facts we get that $Q=\mathscr{A}_{0} \tau$ is a vector subspace of $\Gamma_{0}^{\wedge 1}$, such that $\mathscr{A}_{0} Q \subset Q, Q \mathscr{A}_{0} \subset Q, Q^{*} \subset Q, \Gamma_{\Gamma_{0}} \Phi^{\wedge} Q \subset Q \otimes \mathscr{A}$. We put $S^{\wedge 0}=\mathscr{A}_{0}$, $S^{\wedge 1}=\Gamma_{0}^{\wedge 1} / Q$. The properties of $\Gamma_{0}^{\wedge}$ imply that on the level of $S^{\wedge 0} \oplus S^{\wedge 1}$ all conditions of Definitions 1-2 (for $c=0)$ are satisfied $\left(\wedge, \sigma^{\wedge}, d, *\right.$ are obtained from $\Gamma_{0}^{\wedge 1}$; we use [P 2, Theorem 1.a]). Moreover, (1) gives (13), while (4) yields (15).

Let us define $R_{k m}, r_{k m}, k, m=-1,0,1$, as in the proof of the uniqueness. It can be easily checked that $r_{11}\left(e_{0}\right)=r_{-1-1}\left(e_{0}\right)=1$ and all other $r_{i j}\left(e_{k}\right)$ vanish. The equality

$$
\left[(d \otimes \mathrm{id})\left(\sigma e_{k}\right)\right] \sigma(a)=\sigma^{\wedge}\left(\left(d e_{k}\right) a\right)=\sigma\left(R_{k s}(a)\right)\left[(d \otimes \mathrm{id}) \sigma e_{s}\right]
$$

implies [see (4)]

$$
\left(I \otimes d_{1, m k}\right)\left[\left(R_{m l} \otimes \mathrm{id}\right) \sigma(a)\right]=\sigma\left(R_{k s}(a)\right)\left(I \otimes d_{1, l s}\right),
$$

$a \in \mathscr{A}_{0}, k, l=-1,0,1$. Acting $e \otimes$ id on both sides we get

$$
R_{k s}(a)=d_{1, m k}\left[\left(r_{m l} \otimes \mathrm{id}\right) \sigma(a)\right] w_{l s}
$$

( $w$ was introduced at the beginning of the section). Hence,

$$
R_{n m}\left(e_{j}\right)=r_{k s}\left(e_{i}\right) d_{1, k n} d_{1, i j} w_{s m}, \quad n, m, j=-1,0,1 .
$$

In the following we will need

Definition 3 (cf. [W 5]). We say that $\left(M, \sigma_{M},{ }_{M}\right)$ is a right-covariant $*$-bimodule over $\mathscr{A}_{0}$ iff $M$ is a bimodule over $\mathscr{A}_{0}, \sigma_{M}: M \rightarrow M \otimes \mathscr{A}$ is a linear mapping, $*_{M}: M \rightarrow M$ is an antilinear involution,

$(\mathrm{id} \otimes e) \sigma_{M}=\mathrm{id}, \quad\left(\sigma_{M} \otimes \mathrm{id}\right) \sigma_{M}=(\mathrm{id} \otimes \Phi) \sigma_{M}, \quad\left(\sigma_{M}\right) *_{M}=\left(*_{M} \otimes *\right) \sigma_{M}$

and

$$
\begin{gathered}
\sigma_{M}(\eta a)=\sigma_{M}(\eta) \sigma(a), \quad \sigma_{M}(a \eta)=\sigma(a) \sigma_{M}(\eta), \\
(\eta a)^{*}=a^{*} \eta^{*}, \quad(a \eta)^{*}=\eta^{*} a^{*} \text { for } \eta \in M, \quad a \in \mathscr{A}_{0} .
\end{gathered}
$$

Let $\mathbf{M}=\left(M, \sigma_{M},{ }_{M}\right)$ be a right-covariant $*$-bimodule over $\mathscr{A}_{0}$. We define $\sigma_{M \otimes M}$ and $*_{M \otimes M}$ by

$$
\sigma_{M \otimes M}(m \otimes n)=\sum_{i, j} a_{i} \otimes c_{j} \otimes b_{i} d_{j}
$$

if $\sigma_{M}(m)=\sum_{i} a_{i} \otimes b_{i}, \sigma_{M}(n)=\sum_{j} c_{j} \otimes d_{j}, a_{i}, c_{j} \in M, b_{i}, d_{j} \in \mathscr{A} ;(m \otimes n)^{*_{M} \otimes M}=-n^{*} \otimes m^{*}$, $m, n \in M$. Then $\mathbf{M} \otimes \mathbf{M}=\left(M \otimes M, \sigma_{M \otimes M},{ }_{M}{ }_{M \otimes M}\right)$ is also a right-covariant *-bimodule over $\mathscr{A}_{0}$. Set

$$
M \otimes_{\mathscr{A}_{0}} M=(M \otimes M) / \operatorname{span}\left\{m a \otimes n-m \otimes a n: m, n \in M, a \in \mathscr{A}_{0}\right\} .
$$

We get that $\mathbf{M} \otimes_{\mathscr{A}_{0}} \mathbf{M}=\left(M \otimes_{\mathscr{A}_{0}} M, \sigma_{M \otimes_{\mathscr{A}_{0}} M}, *_{M \otimes_{\mathscr{S}_{0}} M}\right)$ (where $\sigma_{M \otimes_{\mathscr{S}_{0}} M}, *_{M \otimes_{\mathscr{S}_{0}} M}$ are implemented from $\mathbf{M} \otimes \mathbf{M}$ ) is also a right-covariant $*$-bimodule over $\mathscr{A}_{0}$. An element of $M \otimes_{\mathscr{A}_{0}} M$ which is the projection of $m \otimes n \in M \otimes M$ is denoted by $m \otimes_{\mathscr{A}_{0}} n$. 
We return to the proof of existence. We define $S^{\wedge 2}$ as a free left module (over $\mathscr{A}_{0}$ ) with basis $\omega$. We set

$$
\begin{gathered}
b(a \omega)=(b a) \omega, \quad(a \omega) b=(a b) \omega, \\
\sigma^{\wedge}(a \omega)=\sigma(a)(\omega \otimes I), \quad(a \omega)^{*}=-a^{*} \omega, \quad a, b \in \mathscr{A}_{0} .
\end{gathered}
$$

Then $\mathbf{S}^{\wedge 2}=\left(S^{\wedge 2}, \sigma^{\wedge 2}, *\right)$ is a right-covariant $*$-bimodule, which satisfies (9) and (14). We shall also consider right-covariant $*$-bimodule $\mathbf{T}=\mathbf{S}^{\wedge 1} \otimes_{\mathscr{A}_{0}} \mathbf{S}^{\wedge 1}$. Denote $y=q^{-2}\left(1+q^{2}\right)^{-2}\left(q^{6}-1\right)$.

Lemma. There exists a linear mapping $\psi: T \rightarrow S^{\wedge 2}$ such that

$$
\psi\left(a_{k} d e_{k} \otimes_{\mathscr{A}_{0}} b_{l} d e_{l}\right)=a_{k} R_{k m}\left(b_{l}\right)\left(B_{r, m l} e_{r}+y C_{r, m l} \tilde{e}_{r}\right) \omega,
$$

for any $a_{k}, b_{k} \in \mathscr{A}_{0}, k=-1,0,1$.

Proof. a) Let $a_{k} d e_{k}=0$. Then $a_{k}=a \cdot a_{m k} e_{m}, k=-1,0,1$, for some $a \in \mathscr{A}_{0}$. Due to (26), the right-hand side of (31) vanishes.

b) Let $b_{l} d e_{l}=0$. Then $b_{l}=b \cdot a_{j l} e_{j}, l=-1,0,1$, for some $b \in \mathscr{A}_{0}$. The right-hand side of (31) equals

$$
a_{k} R_{k n}(b) a_{j l} R_{n m}\left(e_{j}\right)\left[B_{r, m l} d_{1,0 r}+y C_{r, m l} d_{2,0 r}\right]
$$

(due to [P 2, Eq. (4)], $e\left(\tilde{e}_{r}\right)=\delta_{0 r}$, hence

$$
\tilde{e}_{r}=(e \otimes \mathrm{id}) \sigma \tilde{e}_{r}=e\left(\tilde{e}_{m}\right) d_{2, m r}=d_{2,0 r}, \quad r=-2, \ldots, 2 ;
$$

moreover, $\left.e_{r}=d_{1,0 r}, r=-1,0,1\right)$. But using (30), (2), (18), and (19) one gets

$$
\begin{aligned}
a_{j l} R_{n m}\left(e_{j}\right) B_{r, m l} d_{1,0 r} & =r_{k s}\left(e_{i}\right) a_{j l} d_{1, k n} d_{1, i j}\left(d_{1, b l} w_{b t}\right) w_{s m} B_{r, m t} d_{1,0 r} \\
& =d_{1, k n} r_{k s}\left(e_{i}\right) a_{i b} B_{0, s b}=0
\end{aligned}
$$

(we can set $i=0, a=0, s=0$ ). After an analogous calculation for the second part of (32) we get that the right-hand side of (31) vanishes.

By virtue of a) and b) there exists $\tilde{\psi}: S^{\wedge 1} \otimes S^{\wedge 1} \rightarrow S^{\wedge 2}$ given by

$$
\tilde{\psi}\left(a_{k} d e_{k} \otimes b_{l} d e_{l}\right)=a_{k} R_{k m}\left(b_{l}\right)\left(B_{r, m l} e_{r}+y C_{r, m} \tilde{e}_{r}\right) \omega .
$$

It is easy to check that for any $a \in \mathscr{A}_{0}$ one has

$$
\tilde{\psi}\left(a_{k} d e_{k} a \otimes b_{l} d e_{l}\right)=\tilde{\psi}\left(a_{k} d e_{k} \otimes a b_{l} d e_{l}\right) .
$$

Therefore, the desired mapping $\psi$ exists.

We return once again to the proof of existence. In the following we shall study the properties of $\psi$. We set

$$
J_{l}=a_{s m} e_{s}\left[B_{r, m l} e_{r}+y C_{r, m l} \tilde{e}_{r}\right], \quad l=-1,0,1 .
$$

Using (17) and [P 2, Eq. (5)] we obtain $\sigma J_{l}=J_{k} \otimes d_{1, k l}$, hence $J_{l}=(e \otimes$ id $) \sigma J_{l}$ $=e\left(J_{k}\right) d_{1, k l}$. But $e\left(J_{k}\right)=a_{0 m}\left[B_{0, m k}+y C_{0, m k}\right]=0$ (we can put $m=0$ ). Therefore, $J_{l}=0, l=-1,0,1$. That and (31) prove that

$$
\psi\left(d e_{k} \otimes_{\mathscr{A}_{0}} d e_{l}\right)=\left(B_{r, k l} e_{r}+y C_{r, k l} \tilde{e}_{r}\right) \omega .
$$

Set $\quad A=a_{k l} d e_{k} \otimes_{\mathscr{A}_{0}} d e_{l}, \quad B_{r}=b_{k l, r} d e_{k} \otimes_{\mathscr{A}_{0}} d e_{l}, \quad r=-1,0,1, \quad C_{r}=c_{k l, r} d e_{k} \otimes_{\mathscr{A}_{0}} d e_{l}$, $r=-2, \ldots, 2$. Using (20), we obtain

$$
\psi(A)=0, \quad \psi\left(B_{r}\right)=e_{r} \omega, \quad \psi\left(C_{r}\right)=y \tilde{e}_{r} \omega,
$$

for all possible $r$. 
By virtue of (31) $\psi(a \eta)=a \psi(\eta)$ for all $a \in \mathscr{A}_{0}, \eta \in T$. Therefore, in order to prove $\psi(\eta a)=\psi(\eta) a\left(a \in \mathscr{A}_{0}, \eta \in T\right)$, it suffices to check it for $\eta=d e_{r} \otimes_{\mathscr{A}_{0}} d e_{l}, a=e_{m}$, $r, l, m=-1,0,1$. Then $\eta a=\left(d e_{r} \otimes_{\mathscr{A}_{0}} d e_{l}\right) e_{m}=R_{r a}\left(R_{l b}\left(e_{m}\right)\right) d e_{a} \otimes_{\mathscr{A}_{0}} d e_{b}$. Due to (30), $\sigma R_{l b}\left(e_{m}\right)=R_{c p}\left(e_{n}\right) \otimes d_{1, c l} d_{1, n m} w_{p b}$. Therefore, using (29), we get

$$
R_{r a}\left(R_{l b}\left(e_{m}\right)\right)=d_{1, s r} d_{1, c l} d_{1, n m} r_{s t}\left(R_{c p}\left(e_{n}\right)\right) w_{p b} w_{t a} .
$$

Now (33) gives $\psi(\eta a)=d_{1, \text { sr }} d_{1, c l} d_{1, n m} G_{s c n} \omega$, where

$$
\begin{aligned}
G_{s c n} & =r_{s t}\left(R_{c p}\left(e_{n}\right)\right) w_{p b} w_{t a}\left(B_{r, a b} e_{r}+y C_{r, a b} \tilde{e}_{r}\right) \\
& =r_{s t}\left(R_{c p}\left(e_{n}\right)\right)\left(B_{0, t p}+y C_{0, t p}\right), \quad s, c, n=-1,0,1 .
\end{aligned}
$$

But, using (24) and (25), we get

$$
\begin{gathered}
r_{11}\left(R_{c-1}\left(e_{n}\right)\right)=-q^{2} B_{-1, c n}+C_{-1, c n} \\
r_{-1-1}\left(R_{c 1}\left(e_{n}\right)\right)=B_{1, c n}+q^{2} C_{1, c n}, \quad c, n=-1,0,1 ;
\end{gathered}
$$

others $r_{s t}\left(R_{c p}\left(e_{n}\right)\right) \quad$ vanish if $t+p=0$. Thus $G_{1-10}=-q^{2}\left(1+q^{2}\right)^{-2}$, $G_{-110}=\left(1+q^{2}\right)^{-2}$, others $G_{s c n}$ vanish. That, [P 2, Eq. (5)] and (34) give

$$
\begin{gathered}
\psi\left(A e_{m}\right)=0=\psi(A) e_{m}, \\
\psi\left(B_{r} e_{m}\right)=e_{r} e_{m} \omega=\psi\left(B_{r}\right) e_{m}, \quad r=-1,0,1, \\
\psi\left(C_{r} e_{m}\right)=y \tilde{e}_{r} e_{m} \omega=\psi\left(C_{r}\right) e_{m}, \quad r=-2, \ldots, 2 .
\end{gathered}
$$

It proves $\psi(\eta a)=\psi(\eta) a$.

In order to prove $\psi\left(\eta^{*}\right)=\psi(\eta)^{*}(\eta \in T)$ it suffices to consider $\eta=A, \eta=B_{r}$, $r=-1,0,1, \eta=C_{r}, r=-2, \ldots, 2$. But then that fact follows from (34), properties of $\mathbf{S}^{\wedge 2}$ and equalities

$$
A^{*}=-A, \quad B_{r}^{*}=-B_{-r}, \quad C_{r}^{*}=-C_{-r} .
$$

Moreover, the equalities (34) prove

$$
(\psi \otimes \mathrm{id}) \sigma_{T}=\sigma^{\wedge 2} \psi
$$

Now, for $\theta, \phi \in S^{\wedge 1}$, we put

$$
\theta \wedge \phi=\psi\left(\theta \otimes_{\mathscr{A}_{0}} \phi\right) \in S^{\wedge 2} .
$$

Moreover, for $n>2$, we set $S^{\wedge n}=\{0\}$. The above data determine $\wedge$ completely. Using the properties of $\psi$ one can check that all conditions of Definitions 1-2 (except of condition 4) are fulfilled. Moreover, (10)-(12) follow from (34).

For $\theta=a_{k} d e_{k} \in S^{\wedge 1}\left(a_{k} \in \mathscr{A}_{0}, k=-1,0,1\right)$, we set $d \theta=d a_{k} \wedge d e_{k} \in S^{\wedge 2}$. By virtue of (10), $d: S^{\wedge 1} \rightarrow S^{\wedge 2}$ is a well defined linear mapping. For $\theta \in S^{\wedge n}, n \geqq 2$, we set $d \theta=0$. Hence the condition 4.d follows and the equation

holds for all $\eta \in S^{\wedge 1}, x \in \mathscr{A}_{0}$.

$$
d(x \eta)=x(d \eta)+d x \wedge \eta
$$

We define $L_{r}, r=-2, \ldots, 2$, by the equation $d d \tilde{e}_{r}=L_{r} \omega$. An easy calculation shows that $e\left(L_{r}\right)=0$, hence

$$
L_{r}=(e \otimes \mathrm{id}) \Phi L_{r}=(e \otimes \mathrm{id})\left(L_{m} \otimes d_{2, m r}\right)=0 .
$$

Therefore, $d d\left(e_{k} e_{m}\right)=0$ for all $k, m=-1,0,1\left(e_{k} e_{m}\right.$ are combinations of (2), (3), and [P 2, Eq. (4)]). It proves

$$
d d x=0, \quad d(\eta x)=(d \eta) x-\eta \wedge d x
$$


for $\eta=d e_{k}, x=e_{m}$. Let $K \subset \mathscr{A}_{0}$ be the set of such $x$, which satisfy (36) for all $\eta \in S^{\wedge 1}$. Using (35), we get $e_{m} \in K, m=-1,0,1$. But it is easy to see that $K$ is an algebra. Therefore, (36) holds for all $\eta \in S^{\wedge 1}$ and $x \in \mathscr{A}_{0}$. The remaining part of condition 4 is easy to check. It proves the existence of $\left(_{\cdot}^{2}\right)$-dimensional exterior algebra on $S_{q 0}^{2}$, $q \in(-1,1) \backslash\{0\}$, invariant w.r.t. $\sigma_{q 0}$.

The remaining statements of the theorem follow from the above proofs.

\section{Differential Operators}

In this section we investigate generalized directional derivatives, corresponding to (2)-dimensional exterior algebras on quantum spheres $S_{q 0}^{2}, q \in(-1,1) \backslash\{0\}$, introduced in Sect. 1. We provide classical $(q \rightarrow 1)$ limits of these derivatives.

Let $\mathbf{S}^{\wedge}$ be $\left({ }^{2}\right)$-dimensional exterior algebra on $S_{q 0}^{2}$, invariant w.r.t. $\sigma_{q 0}$, $q \in(-1,1) \backslash\{0\}$. According to $[\mathrm{P} 1]$, we set $e_{ \pm 1}= \pm i\left(x_{1} \pm i x_{2}\right), e_{0}=2 x_{3}$, i.e. $e_{k}=p_{m k} x_{m}$, for some real numbers $p_{m k}, m=1,2,3, k=-1,0,1$. The relation (2) takes in the language of $x_{k}$ the form $s_{a b} x_{a} x_{b}=I$, where $s_{a b}=p_{a i} a_{i j} p_{b j}, a, b=1,2,3$. Analogously, using our main result, we have that the left module $S^{\wedge 1}$ is generated by $d x_{1}, d x_{2}, d x_{3}$, satisfying a unique constraint $s_{a b} x_{a} d x_{b}=0$. Hence, there exist unique generalized directional derivatives $\widetilde{D}^{k}: \mathscr{A}_{0} \rightarrow \mathscr{A}_{0}, k=1,2,3$, such that

$$
d a=\widetilde{D}^{k}(a) d x_{k}, \quad \tilde{D}^{k}(a) x_{k}=0, \quad a \in \mathscr{A}_{0} .
$$

Analogously, there exist unique operators $\tilde{G}^{k l}: \mathscr{A}_{0} \rightarrow \mathscr{A}_{0}, k, l=1,2,3$, such that

$$
\left(d x_{k}\right) a=\widetilde{G}^{k l}(a) d x_{l}, \quad \tilde{G}^{k l}(a) x_{l}=0, \quad k=1,2,3, \quad a \in \mathscr{A}_{0} .
$$

Similarly as in [P2, proof of Theorem 3.a] one has

$$
\begin{gathered}
\tilde{D}^{k}(x y)=x \tilde{D}^{k}(y)+\tilde{D}^{l}(x) \tilde{G}^{l k}(y), \quad k=1,2,3, \quad x, y \in \mathscr{A}_{0}, \\
\tilde{G}^{k l}(x y)=\tilde{G}^{k s}(x) \tilde{G}^{s l}(y), \quad k, l=1,2,3, \quad x, y \in \mathscr{A}_{0}
\end{gathered}
$$

(the right-hand sides satisfy the conditions defining the left-hand sides).

It is easy to check that

$$
\begin{gathered}
\tilde{D}^{k}(a)=D^{k}(a)-\left[D^{n}(a) x_{n}\right] s_{i k} x_{i}, \quad k=1,2,3, \quad a \in \mathscr{A}_{0}, \\
\tilde{G}^{k l}(a)=G^{k l}(a)-\left[G^{k n}(a) x_{n}\right] s_{i l} x_{i}, \quad k, l=1,2,3, \quad a \in \mathscr{A}_{0},
\end{gathered}
$$

where $D^{k}, G^{k l}$ were introduced in [P 2, Eq. (21) and proof of Theorem 3.a] (their defining relations hold also in $S^{\wedge 1}$, which is a projection of $\Gamma_{0}^{\wedge 1}$, see the proof of existence).

According to [P 2, Theorem 3.a and its proof $]$,

$$
\lim D^{k}=\partial^{k}-(1 / 2) x_{k} \bar{\Delta}, \quad \lim G^{k l}=\delta_{k l}-x_{l} \hat{\partial}^{k},
$$

$k, l=1,2,3$. Therefore, using (37)-(38), one gets

$$
\lim \widetilde{D}^{k}=\partial^{k}=\widetilde{D}_{\left.\right|_{q=1}}, \quad \lim \tilde{G}^{k l}=\delta_{k l}-R^{-2} x_{k} x_{l}=\widetilde{G}_{\mid q=1}^{k l},
$$

where $R=1 / 2$ (for $q=1 \tilde{D}^{k}, \tilde{G}^{k l}$ are defined in the same way as above). Similarly as in [P 2, Sect. 2], the question of finding all right-invariant differential operators remains open. 
Acknowledgements. I am very indebted to Prof. S. L. Woronowicz for his encouragement to study exterior forms on quantum spheres. I am also grateful to Prof. H. Araki for his kind hospitality in RIMS. My stay here is supported by a Monbusho Fellowship. I thank Prof. P. Kulish and Dr. A. Schnizer for their remarks concerning the paper.

\section{References}

[C] Connes, A.: Non-commutative differential geometry. Publ. IHES 62, 41-144 (1985)

[CSSW] Carow-Watamura, U., Schlieker, M., Scholl, M., Watamura, S.: Tensor representation of the quantum group $S L_{q}(2, \mathrm{C})$ and quantum Minkowski space. Z. Phys. C Particles and Fields 48, 159-165 (1990)

[CSW] Carow-Watamura, U., Schlieker, M., Watamura, S.: $\mathrm{SO}_{q}(N)$ covariant differential calculus on quantum space and quantum deformation of Schrödinger equation. $Z$. Phys. C - Particles and Fields 49, 439-446 (1991)

[M] Manin, Yu.I.: Some remarks on Koszul algebras and quantum groups. Ann. Inst. Fourier 37, 4, 191-205 (1987)

[Mau] Maumary, S.: $C^{*}$-surgery on $C^{*}$-manifolds. Talk at Department of Mathematics, Kyoto University, 1991

[P 1] Podleś, P.: Quantum spheres. Lett. Math. Phys. 14, 193-202 (1987)

[P 2] Podleś, P.: Differential calculus on quantum spheres. Lett. Math. Phys. 18, 107-119 (1989)

[P 3] Podles, P.: Quantum spaces and their symmetry groups. Ph.D. Thesis, Warsaw University, 1989 (in Polish)

[P 4] Podleś, P.: Differential calculus on quantum spheres. RIMS Kokyuroku Series, No. 751, May 1991

[P 5] Podlés, P.: Symmetries of quantum spaces. Subgroups and quotient spaces of quantum $S U(2)$ and $S O(3)$ groups. (in preparation)

[PW] Podleś, P., Woronowicz, S.L.: Quantum deformation of Lorentz group. Commun. Math. Phys. 130, 381-431 (1990)

[RTF] Reshetikhin, N.Yu., Takhtadzyan, L.A., Faddeev, L.D.: Quantization of Lie groups and Lie algebras. Leningrad Math. J. 1, 1, 193-225 (1990); Russian original: Algebra i analiz 1 (1989)

[W 1] Woronowicz, S.L.: Pseudospaces, pseudogroups and Pontryagin duality. Proceedings of the International Conference on Mathematics and Physics, Lausanne 1979, Lecture Notes in Physics Vol. 116. Berlin, Heidelberg, New York: Springer 1980, pp. 407-412

[W 2] Woronowicz, S.L.: Twisted $S U(2)$ group. An example of a non-commutative differential calculus. Publ. RIMS, Kyoto Univ. 23, 117-181 (1987)

[W 3] Woronowicz, S.L.: Compact matrix pseudogroups. Commun. Math. Phys. 111, 613-665 (1987)

[W 4] Woronowicz, S.L.: Tannaka-Krein duality for compact matrix pseudogroups. Twisted $S U(N)$ groups. Invent. Math. 93, 35-76 (1988)

[W 5] Woronowicz, S.L.: Differential calculus on compact matrix pseudogroups (quantum groups). Commun. Math. Phys. 122, 125-170 (1989)

[WZ] Wess, J., Zumino, B.: Covariant differential calculus on the quantum hyperplane. Nucl. Phys. B (Proc. Suppl.) 18 B, 302-312 (1990) 
